\title{
The Extreme-Energy Events Project: a bridge between school and professional research
}

\author{
Silvia Pisano for the EEE Collaboration ${ }^{a, b, *}$ \\ ${ }^{a}$ Museo storico della fisica e Centro di studi e ricerche "Enrico Fermi, \\ Via Panisperna, 89a, 00184 Roma, Italy \\ ${ }^{b}$ INFN - Laboratori Nazionali di Frascati, \\ Via Enrico Fermi 54 (già 40) - 00044 Frascati (Roma) Italy \\ E-mail: silvia.pisano@lnf.infn.it
}

The Extreme-Energy Events (EEE) Experiment is a cosmic ray observatory based on a network of detecting stations distributed over the Italian territory and at CERN. A station of the network, called "telescope", consists of three superimposed Multi-gap Resistive Plate Chambers (MRPCs), each one covering a surface of about $1.5 \mathrm{~m}^{2}$, used with the same technology as the time-of-flight detector of the ALICE experiment at CERN-LHC. Data are collected and sent in real time to the INFN-CNAF computer center for reconstruction and analysis. The large area covered by the network, ranging from Southern Italy up to the CERN laboratories, was achieved with the decision to install the detectors inside high schools, involving students and teachers in a modern experiment within a unique program. This very coexistence of scientific activity and outreach represents the uniqueness of the EEE Project. The outreach programme is articulated in several initiatives, each encoding the different aspects of the research activity normally expected in a highenergy physics experiment. Students are involved in detector construction at CERN, installation in school, and in the commissioning of the station when data taking starts. Once the detector reaches a steady working regime, students are requested to monitor on a daily basis the performance of the telescope and report any failure. In parallel to the hardware-related operations, students learn how to perform the analysis of EEE data under the supervision of their teachers and of the EEE researchers, supporting the scientific output of the experiment. Every month students report progress and issues in a dedicated online meeting open to all schools and to the EEE researchers. Beside this monthly appointment, in the pre-COVID era an in-person meeting was taking place twice per year, hosted by the Ettore Majorana Foundation and Centre for Scientific Culture in Erice or by a school or institution involved in the project. During a three-day, students attend masterclasses and take part in measurement campaigns, disseminating their results by submitting contributions to important outreach-oriented journals (such as the Italian Giornale di Fisica).

The Ninth Annual Conference on Large Hadron Collider Physics - LHCP2021

7-12 June 2021

Online

\footnotetext{
${ }^{*}$ Speaker
} 


\section{Introduction}

The Extreme Energy Events (EEE) Projects [1] aims at the observation and reconstruction of high-energy cosmic ray events through the detection of secondary showers on the earth surface. It is a large array of particle detectors installed in Italian high-school buildings and laboratories, and spans an area of more than $10^{5} \mathrm{~km}^{2}$, from CERN to Sicily. It is based on a network of Multigap Resistive Plate Chamber (MRPC) telescopes, inspired by the ones composing the timeof-flight system of the ALICE detector at CERN. While being a scientific project, the main mission of the EEE Project is educational, providing a full research experience to high-school students. EEE Project is a collaboration between Centro Fermi [2] and the National Institute for Nuclear Physics (INFN - Istituto Nazionale di Fisica Nucleare) [3]. The Collaboration is composed of dozens of professional researchers and research associates, that operate the network organizing and monitoring the data taking and performing data analysis. However, in addition to the scientific staff, a major role is played by students from more than 100 Italian High Schools that take part in almost all the aspects of the experiment scientific life. The excellent performance of the detectors allowed the EEE Collaboration to perform relevant measurements in the field of cosmic-ray physics, based on the detection of the secondary muon component. In particular, beyond the measurements devoted to the observation of the Extensive Air Showers (EASs) [4, 5], studies have been devoted to the search of possible anisotropies at the sub-TeV scale [6], to the analysis of solar phenomena [7, 8], to the measurement of upward-going particles [9], as well as the search of long distance correlations among detectors located from few to hundred kilometers apart [10, 11]. In addition, different performance studies have been carried out [12, 13], and a dedicated simulation tool has been developed [14].

\section{The network}

Detectors are distributed overall Italy (plus 2 stations located at CERN), spanning $10^{\circ}$ in latitude and $11^{\circ}$ in longitude. Data from each station are synchronized through the GPS system and sent to the INFN CNAF in Bologna for track reconstruction [15]. In those sites where more than one telescope is installed in the same metropolitan area, tracks from different telescopes are combined to identify showers of secondary cosmic rays, with a time synchronization realized through a GPS-based system. Since March 2019 a collaboration with the Italian Istituto Nazionale di Ricerca Metrologica (INRIM) [16] is active to develop a new synchronization strategy. A station is equipped with three MRPCs, each having a sensitive area of about $80 \times 160 \mathrm{~cm}^{2}$. The MRPCs are a stack of glass plates, separated from each other with fishing line, enclosing six $300 \mu \mathrm{m}$ gaps where the gas circulates, as shown in Fig.1. Chambers are powered by high voltage (around $20 \mathrm{kV}$ ), applied only to the external glasses, that are coated with resistive paint. In between the external glasses, a proper gas mixture at atmospheric pressure provides the active medium. In the last years, a campaign toward the identification of a new, ecological mixture - in compliance with the new regulations by EU - has started [17]. The readout panel is split in 24 copper strips. The anode and cathode strips collect the signals induced by the particles, providing position information along one direction. The information along the other coordinate is obtained by measuring the time delay between the signals arriving at the two ends of a strip. 


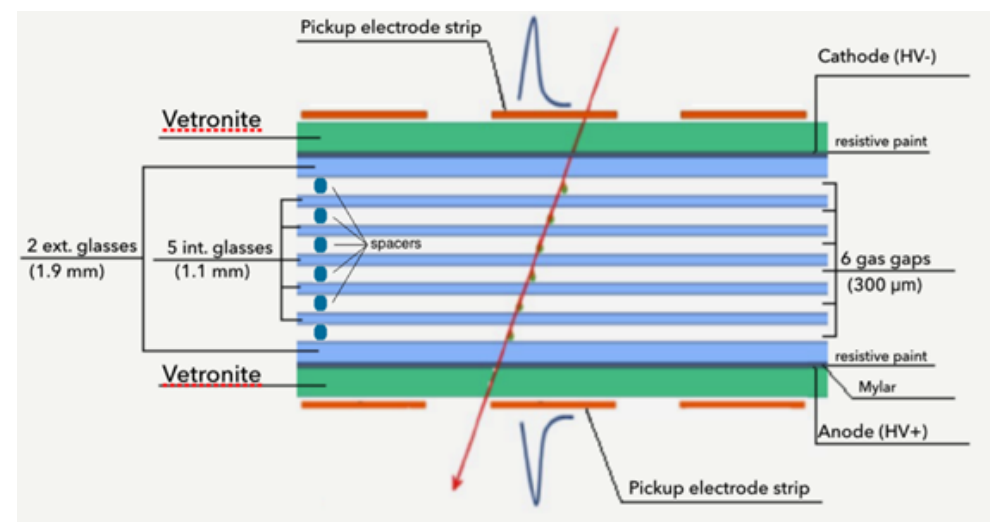

Figure 1: Inner structure of a MRPC.
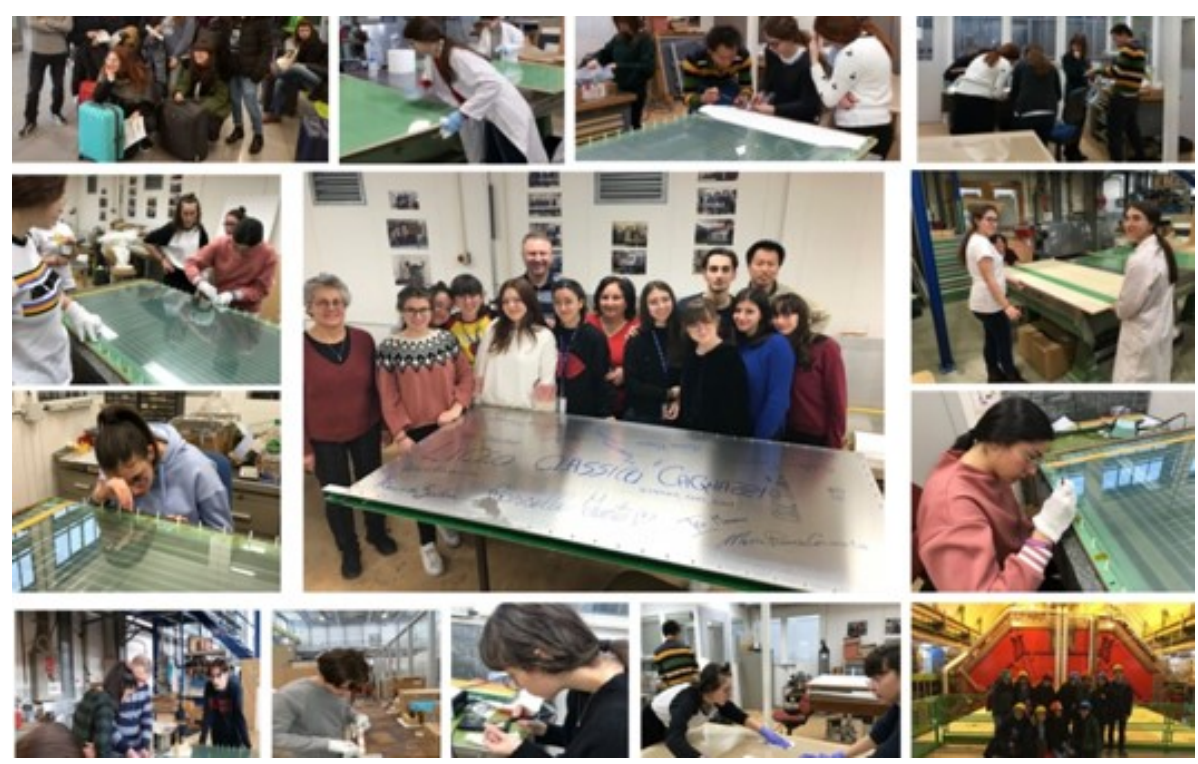

Figure 2: High-school students at CERN taking part in the construction of a MRPC for a EEE station.

\section{Outreach}

The EEE Project represents a perfect environment to introduce students to scientific research. In fact, students are involved in all the different aspects of the project: the detector construction at CERN, where students spend a week and, under the supervision of EEE researchers, actively participate in the detector assembly and test (see Fig.2), the commissioning of the station when it is delivered to their school, the analysis of the collected data. In particular, students are responsible for the monitoring activities, filling, on a daily basis, a check-list reporting the main information on the data acquisition conditions, as weather parameters (pressure, temperature), acquisition rate, high-voltage settings and currents in the chambers. Furthermore, on the local acquisition system different observables are plotted for every run and checked by students to test the quality of the collected data. In case of issues, students and professors refer to a local referent - an EEE researcher 


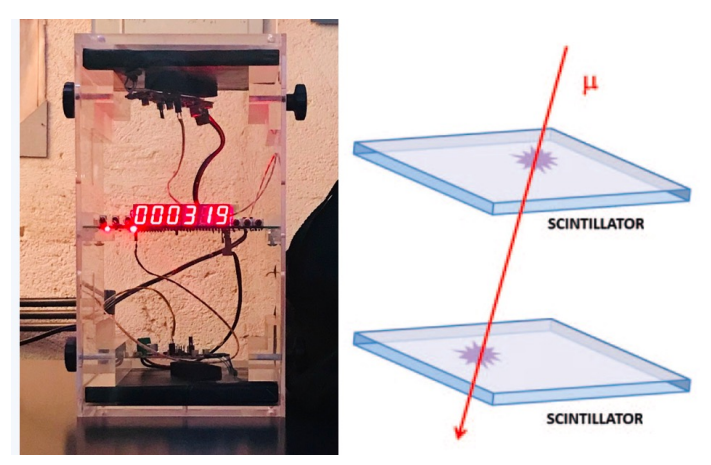

Figure 3: Left: the Cosmic Box used to carry out the measurement of the cosmic ray variation with altitude. Right: working principle of a Cosmic Box.

- who provides instructions on possible actions to be taken. In order to keep the students up to date on the network status and to provide them the possibility to discuss their activities, the EEE Collaboration, on a monthly basis, organizes a Run Coordination Meeting with the schools where students can present the status of their station and the ongoing data analyses, receiving inputs from the scientific staff. Beyond the presentations by students, during the monthly meetings lectures on cosmic rays and modern physics are organized, together with masterclasses dedicated to relevant tools and topics for data analysis (ROOT, statistics etc). In addition to these meetings, twice per year (in the pre-COVID era) conferences were organized with the EEE schools to discuss the status of the experiment. These conferences, that usually last three days, are hosted by the Ettore Majorana Foundation and Centre for Scientific Culture in Erice: students present their work, take part in masterclasses and participate in measurement campaigns. As to the latter, one of these campaigns was devoted to the measurement of the muon flux at different altitudes, which led to an educational publication [18], while another, whose goal was to replicate the Eratostene experiment, produced an estimation of the Earth radius [19]. For the first measurement, the so-called Cosmic Box, a portable, scintillator-based detector built by EEE students, has been used (see Fig.3). In addition to these initiatives, other projects involving students are active within the EEE Collaboration. Among them, in the PolarQUEEEst experiment [20] - inspired by the old-fashioned scientific expeditions - a boat sailed through the Svalbard archipelago, getting to a latitude of $82^{\circ} 07^{\prime} \mathrm{N}$, performing a set of multidisciplinary experiments (PCBs, polychlorinated biphenyls, micro- and nano-plastics (CNRISMAR), polar drones). The EEE Collaboration was responsible for the measurements of the cosmic ray flux up to the far North latitudes, and, to this end, a portable cosmic ray detector, POLA, was designed [21-23], with students actively participating in the detector construction.

\section{Summary}

The EEE Project, in its two-fold nature of a scientific experiment and an outreach initiative, offers a unique environment for initiating students to scientific research, directly involving them in all the different activities of the experiment, from the detector construction at CERN to the data taking and analysis. Up to now, 200 MRPCs have been built and installed in Italian high-schools, with the network still expanding and welcoming new schools. 


\section{References}

[1] EEE web site: https://eee.centrofermi.it/

[2] https://cref.it/en/

[3] https://home.infn.it/en/

[4] EEE Collaboration, First detection of extensive air showers with the EEE experiment, Nuovo Cimento 125 B (2010) 243.

[5] EEE Collaboration, Time Correlation measurements from extensive air showers detected by the EEE telescopes, Eur. Phys. J. Plus (2013) 128, 148.

[6] EEE Collaboration, Looking at the sub-TeV sky with cosmic muons detected in the EEE MRPC telescopes, Eur. Phys. J. Plus 130 (2015) 187.

[7] EEE Collaboration, Observation of the February 2011 Forbush decrease by the EEE telescopes, Eur. Phys. J. Plus (2011) 126, 61.

[8] EEE Collaboration, Results from the observation of Forbush decreases by the Extreme Energy Events experiment, Proceedings of the ICRC2015 Conference, PoS(ICRC2015)097.

[9] EEE Collaboration, A study of upward-going particles with the EEE telescopes, NIM A 816 (2016) 142.

[10] EEE Collaboration, Time and orientation long distance correlations between extensive air showers detected by the MRPC telescopes of the EEE Project, Nuovo Cimento C40(2017)196.

[11] EEE Collaboration, Search for long distance correlations between extensive air showers detected by the EEE network, Eur. Phys. J. Plus (2018) 133: 34.

[12] EEE Collaboration, Performance of a six gap MRPC built for large area coverage, NIM A 593 (2008) 263.

[13] EEE Collaboration, Recent results and performance of the multi-gap resistive plate chambers network for the EEE Project, JINST 11 (2016) C11005.

[14] EEE Collaboration, The cosmic muon and detector simulation framework of the extreme energy events (EEE) experiment, Eur. Phys. J. C (2021) 81:464.

[15] E-log web site: https://iatw.cnaf.infn.it/eee/elog/

[16] https://www.inrim.eu/

[17] EEE Collaboration, Test of new eco-gas mixtures for the multigap resistive plate chambers of the EEE project, NIM A 936 (2019) 493. 
[18] EEE Collaboration, FISICA PER TUTTI - Come varia il flusso dei raggi cosmici con la quota? Basta chiederlo agli studenti del progetto EEE - How does cosmic ray flux vary with altitude? Let's ask it to EEE project students, Giornale di Fisica, VOL. LIX, N. 3, Luglio - Settembre 2018.

[19] EEE Collaboration, Gli studenti del progetto EEE sulle orme di Eratostene per la misura del raggio della Terra, Giornale di Fisica 60 (2019) 107.

[20] Polarquest 2018 web site: http://www.polarquest2018.org/

[21] R. Nania and O. Pinazza, Measuring cosmic ray showers near the North Pole with the Extreme Energy EventsProject, Nuovo Sagg. 34 (2018) 27.

[22] M. Abbrescia et al. (EEE collaboration), Results from the PolarquEEEst missions, J. Phys.: Conf. Ser. 1561 012001, 2020, doi:10.1088/1742-6596/1561/1/012001

[23] M. Abbrescia et al. (EEE collaboration), New high precision measurements of the cosmic charged particle rate beyond the Arctic Circle with the PolarquEEEst experiment. Eur. Phys. J. C (2020) 80: 665 https://doi.org/10.1140/epjc/s10052-020-8213-2

\section{Full Authors List: EEE Collaboration}

M. Abbrescia ${ }^{1,2}$, C. Avanzini ${ }^{3}$, L. Baldini ${ }^{3,4}$, R. Baldini Ferroli ${ }^{5}$, G. Batignani ${ }^{3,4}$, M. Battaglieri ${ }^{6,7}$, S. Boi ${ }^{8,9}$, E. Bossini ${ }^{3,4}$, F. Carnesecchi ${ }^{10,11}$, C. Cicalò ${ }^{9}$, L. Cifarelli ${ }^{10,11}$, F. Coccetti ${ }^{12}$, E. Coccia ${ }^{13}$, A. Corvaglia ${ }^{14}$, D. De Gruttola ${ }^{15,16}$, S. De Pasquale ${ }^{15,16}$, F. Fabbri ${ }^{5}$, L. Galante ${ }^{17,18}$, M. Garbini ${ }^{10,12}$, G. Gemme ${ }^{6}$, I. Gnesi ${ }^{12,19}$, S. Grazzi ${ }^{6,20}$, D. Hatzifotiadou ${ }^{10,21}$, P. La Rocca ${ }^{22,23}$, Z. Liu ${ }^{24}$, G. Mandaglio ${ }^{20,23}$, G. Maron ${ }^{25}$, M. N. Mazziotta ${ }^{2}$, A. Mulliri ${ }^{8,9}$, R. Nania ${ }^{10}$, F. Noferini ${ }^{10}$, F. Nozzoli ${ }^{26}$, F. Palmonari ${ }^{10,11}$, M. Panareo ${ }^{14,27}$, M. P. Panetta ${ }^{12,14}$, R. Paoletti ${ }^{3,28}$, C. Pellegrino ${ }^{25}$, O. Pinazza ${ }^{10}$, C. Pinto ${ }^{22,23}$, S. Pisano ${ }^{5,12}$, F. Riggi ${ }^{22,23}$, G. C. Righini ${ }^{29}$, C. Ripoli ${ }^{15,16}$, M. Rizzi ${ }^{2}$, G. Sartorelli ${ }^{10,11}$, E. Scapparone ${ }^{10}$, M. Schioppa $^{19,30}$, A. Scribano ${ }^{28}$, M. Selvi $^{10}$, G. Serri ${ }^{8,9}$,

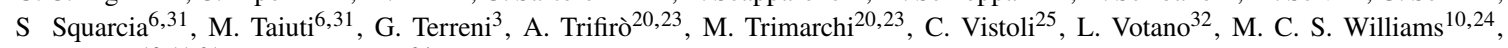
A. Zichichi ${ }^{10,11,21}$ and R. Zuyeuski ${ }^{24}$

${ }^{1}$ Dipartimento Interateneo di Fisica, Università di Bari, Bari, Italy. ${ }^{2}$ INFN Sezione di Bari, Bari, Italy. ${ }^{3}$ INFN Sezione di Pisa, Pisa, Italy. ${ }^{4}$ Dipartimento di Fisica, Università di Pisa, Pisa, Italy. ${ }^{5}$ INFN Laboratori Nazionali di Frascati, Frascati (Rome), Italy. ${ }^{6}$ INFN Sezione di Genova, Genova, Italy. ${ }^{7}$ Thomas Jefferson National Accelerator Facility, Newport News, VA, USA. ${ }^{8}$ Dipartimento di Fisica, Università di Cagliari, Cagliari, Italy. ${ }^{9}$ INFN Sezione di Cagliari, Cagliari, Italy. ${ }^{10}$ INFN Sezione di Bologna, Bologna, Italy. ${ }^{11}$ Dipartimento di Fisica ed Astronomia, Università di Bologna, Bologna, Italy. ${ }^{12}$ Museo Storico della Fisica e Centro Studi e Ricerche "E. Fermi", Rome, Italy. ${ }^{13}$ Gran Sasso Science Institute, LAquila, Italy. ${ }^{14}$ INFN Sezione di Lecce, Lecce, Italy. ${ }^{15}$ Dipartimento di Fisica, Università di Salerno, Salerno, Italy. ${ }^{16}$ INFN Gruppo Collegato di Salerno, Salerno, Italy. ${ }^{17}$ Dipartimento di Scienze Applicate e Tecnologia, Politecnico di Torino, Torino, Italy. ${ }^{18}$ INFN Sezione di Torino, Torino, Italy. ${ }^{19}$ INFN Gruppo Collegato di Cosenza, Cosenza, Italy. ${ }^{20}$ Dipartimento di Scienze Matematiche e Informatiche, Scienze Fisiche e Scienze della Terra, Università di Messina, Messina, Italy. ${ }^{21}$ CERN, Geneva, Switzerland. ${ }^{22}$ Dipartimento di Fisica e Astronomia, Università di Catania, Catania, Italy. ${ }^{23}$ INFN Sezione di Catania, Catania, Italy. ${ }^{24}$ ICSC World laboratory, Geneva, Switzerland. ${ }^{25}$ INFN-CNAF, Bologna, Italy. ${ }^{26}$ INFN Trento Institute for Fundamental Physics and Applications, Trento, Italy. ${ }^{27}$ Dipartimento di Matematica e Fisica, Università del Salento, Lecce, Italy. ${ }^{28}$ Dipartimento di Scienze Fisiche, della Terra e dell'Ambiente, Università di Siena, Siena, Italy. ${ }^{29} \mathrm{CNR}$ Istituto di Fisica Applicata "Nello Carrara", Sesto Fiorentino (Florence), Italy. ${ }^{30}$ Dipartimento di Fisica, Università della Calabria, Rende (Cosenza), Italy. ${ }^{31}$ Dipartimento di Fisica, Università di Genova, Genova, Italy. ${ }^{32}$ INFN, Laboratori Nazionali del Gran Sasso, Assergi (L'Aquila), Italy. 